\title{
The design and manufacture of atmospheric plasma jet surgical handpiece
}

\author{
Hamed Bagheri', Reza Reiazi2,*, Mohammad Kasaie ${ }^{3}$, Hosein Mootabian ${ }^{4}$
}

\begin{abstract}
Background: Atmospheric plasma jet has different medical applications due to its low temperature at room temperature. In recent years, the effect of nonthermal plasmas on cancer cells has been studied, and it has been shown that this type of plasma has anti-proliferative effects on cancer cells.

Objectives: To design a plasma jet handpiece, which can be used in cutting operations in less bleeding surgery, eliminating cancer cells without damage to healthy cells and reducing the duration of wound healing.

Methods: The plasma handpiece simply consists of a nozzle body and two cathode and anode electrodes and a fully insulated body against heat and high voltage. Argon is introduced into the handpiece, and by plasma treatment, it is used for special purposes. Each piece was made according to its own manufacturing process and by assembling; the final product of the atmospheric plasma jet handpiece was ready for testing. The jet pipeline was then tested, and the effective parameters were examined.

Results: The cold atmospheric plasma jet length depends on factors such as power supply, applied voltage, gas flow rate and the distance between the electrodes. The results showed with increasing velocity, the flame and jet lengths decreased greatly due to high losses of plasma, including ions and electrons. Also with increasing the velocity of argon gas, its concentration decreased.

Conclusions: It is concluded that the performance of the proposed design is successful. The advantages include low-cost manufacturing, highly stable performance, and low erosion and can be considered for future development.
\end{abstract}

Keywords: cold atmospheric plasma; plasma; plasma jet; surgical handpiece

Nowadays, with the appearance of new methods in curative medicine, increasing the efficiency and reducing the cost of energy and technology, both medical and nonmedical, has become the focus of interest. Plasma science is receiving increasing interest from the researchers in these areas. The unique nature of plasma has made it widely used in industry and medicine [1]. Plasma is one of the four main phases of a matter; in other words, quasi-neutral gas of charged particles provides collective behavior. The other three phases are solid, liquid, and gas. The term "plasma" refers to ionized gas, all or a significant portion of its atoms lost one or more electrons and turned into positive ions [2].

The passage of electric current from inside an ionized gas leads to a phenomenon called gaseous discharges. This

*Correspondence to: Reza Reiazi, Department of Medical Physics, School of Medicine, Iran University of Medical Sciences, Tehran 1449614535, Iran, e-mail: Reiazi.r@iums.ac.ir

'Radiation Sciences Research Center, Aja University of Medical Sciences, Tehran, Iran

${ }^{2}$ Departmen of Medical Physics, School of Medicine, Iran University of Medical Sciences, Tehran 1449614535, Iran

${ }^{3}$ Department of Mechanical Engineering, Faculty of Engineering, Khajeh Nasir Toosi University of Technology, Tehran 1969764499, Iran

${ }^{4}$ Medical Radiation, Science and Research Branch, Islamic Azad University, Tehran 1477893855, Iran

¿ Open Access. ๑ 2019 Bagheri et al., published by Sciendo. (c) BY-Nc-ND This work is licensed under the Creative Commons Attribution NonCommercial-NoDerivatives 4.0 License. 
phenomenon is the most common method of plasma production. At present, the focus has been given on atmospheric pressure plasma due to its simplicity and low cost than lowpressure plasma [3].

This type of plasma is non-equilibrium; in other words, there is no local thermodynamic equilibrium and content of hot electrons and cold ions and neutral species in the room temperature range [4]. From a macroscopic point of view, this is cold plasma. The importance of cold atmospheric pressure air plasma makes it possible to use in the processing of materials susceptible to heat and living tissue in the industry and medicine [5]. One type of cold atmospheric pressure plasma is plasma jet. In the contact of plasma with the surface of the material, energy is transferred from the plasma and secondary reactions occur at the surface of the material. Plasma surgery techniques, such as coagulation or withdrawal, are primarily based on the deadly effects of plasma on living systems. To achieve the selective effects and avoid the specific hazards, plasma torches should include characteristic components, and it is necessary to know how to control them by external work parameters [6].

Plasma jets include a nozzle with one or two electrodes. The plasma is generated inside the nozzle and transmitted by a gas stream to the bodies. Principally, the differences between all these plasma jets are in the configuration of the electrode, electrode material, the type of gas, frequency, and the amount of applied voltage. The plasma handpiece simply includes a plasma jet that can be taken by the hand. The gas is converted to plasma by passing through the electrodes and is used for various applications [7]

The advantage of plasma with low temperature is the high UV intensity. This radiation has a high microbicidal effect. Many reactions occur in the plasma due to different mechanisms of sterilization. These reactions include ultraviolet irradiation, reactive species, and the eruption of charged particles and spot heat distribution. The degree of penetration of these rays is limited [8]. The plasma technology is a desirable method for modifying surface properties. About the plasma advantage compared to ultraviolet ray generators, gas can penetrate the gaps. The potential benefits of non-heat plasma technology are that ultraviolet radiation, radicals, and other reactive species can affect microbes from anywhere in the plasma. Cold plasma produces high microbial effects by producing ultraviolet rays, causing small chemical changes and can be used in the food industry [9].

Atmospheric plasma jet has different medical applications due to its low temperature at room temperature. The plasma jet interaction with cells and living tissues of the body can be utilized in procedures such as sterilization, wound healing, and cancer treatment. In recent years, the effect of nonthermal plasmas on cancer cells has been studied, and it has been shown that this type of plasma has anti-proliferative effects on cancer cells [10].

\section{Materials and methods}

Designing is known for obtaining optimal solutions for the invention of industrial machines and machines. Design methods are done in a systematic method, and finding the solutions does not occur accidentally.

Despite the widespread use of torches, the process and arc limitations are considered major issues for its application. One of the main factors for this limitation is the lack of recognition and control of the arc dynamics inside the torch and the anode corrosion effect on plasma jet forces. The presence of geometry and symmetric boundary conditions, the gas flow is an intrinsic three-dimensional. Moreover, any arc movement will have a significant effect on the torch and jet output flow.

The arc dynamics resulting from the balancing of the drag force generated from the interactions of the incoming gas flow along the arc and the Lorentz electromagnetic force generated from the local curvature of the arc is continuously displaced in the anode wall. The arc length is proportional to the number of voltage fluctuations.

$$
\overrightarrow{\mathrm{F}}=\rho \overrightarrow{\mathrm{E}}+\overrightarrow{\mathrm{J}} \times \overrightarrow{\mathrm{B}}
$$

It can be said that there is a direct relationship between the flow density and the Lorentz force. The generated arc is constantly inclined to reshape and reconnect, and one of its causes is the presence of Lorentz force. This force in the case of local arc curvature plays an important role. Because of the diverse shape of the arc, the Lorentz force creates an angular momentum that agrees or disagrees with arc coupling. Paschen's law about the electrical discharge of gases, which states the necessary voltage for the electrical breakdown of a gas placed in a uniform field, is a nonlinear function of the gas pressure and the distance between the electrodes.

$$
V_{b}=f(p . d)
$$

Paschen's law curve determines that the voltage breakdown of a given gas is placed at a distance between the electrodes of a uniform field with a specific material, which is a function of the gas pressure and the distance between the electrodes [11]. The validity of Paschen's law is shown in a wide range of $\mathrm{PD}$ values. Nevertheless, in relatively large values of PD, it is seen, in some cases, that the voltage breakdown is lower in the same values of PD with larger distance. On the other hand, at very low pressures, the invalidity of Paschen's law has been observed. In these pressures, the voltage breakdown does not depend on the characteristics of the gas but depends on the purity and properties of the electrodes. The 
plasma torch is the most critical element of plasma-processing equipment. The design of electrodes is the main factor in the performance of most torches, measuring current and operating power over its lifetime. It is difficult to describe the arc and electrode interactions due to different physical phenomena within a range of parameters, such as in various pressures, currents, and densities. Different structures of the electrode are, therefore, used for various applications [12]. The thermionic cathodes include a wider range of arc flows, and it preferred the cold cathode in designs due to the dominance of field emission. As can be seen in Figure 1, the cathode is placed in the plasma jet as a copper rod at an adjustable distance from the annular anode.

From the material behavior's point of view, the anode should be selected from the kind of electron absorber to allow for continuity of flow and cathode generates the additional electrons for the arc. The thermionic cathodes provide hightemperature electrons for producing a large number of electron emissions. Usually, strengthen materials are required in the form of bars, and materials with a lower functionality are added to increase the electron emission in lower temperatures of cathodes. Experimental results indicate that the geometry of electrodes can significantly affect the erosion rate and arc stability [13]. Also, electrodes with an acute head shape can suitably affect the stable discharge. The nozzle is essentially a part of a plasma torch with a high heat load. The nozzle is exposed to the hot plasma, radiation, Joule heating, ion bombardment, and also under electric arc occurrence. The electrode is cooled by the convection, while the nozzle is heated by the convection. When the cold nonplasma gas flows are passed through the cathode and entered into the ionized electrical arc, it is heated intensely, and this hot plasma gas flow with a convective phenomenon exited from the crater the nozzle [14].
The high-temperature plasma flow heats the anode by the radiation. However, this heating effect is usually negligible than other factors. Also, anodic hot spots can radiate the nozzle's heat to a colder gas around the nozzle. Joule heating is an important source of anode heating, but not as much as the heating effect in the cathode. The most important sources of heat transfer to the anode are ion bombardment and electric arc impact. Similar to the cathode that absorbs positive ions and converts their kinetic energy to heat energy, the heat is generated in the nozzle that absorbs the negative ions. The nozzle is cooled by the convection phenomenon, thermal conductivity, and radiation [15].

\section{Results}

By finalizing the design, all parts were made. The argon as suitable gas for the plasma process is selected due to ineffectualness, tastelessness, and inodorous. The argon is very neutral and not combined with any other gas and dissolved in the water rarely. In addition, argon is much more suitable than other gases because it greatly reduces the oxidation process of the cathode and the anode.

\section{The dependence of jet length on applied voltage}

The cold atmospheric plasma jet length depends on factors such as power supply, applied voltage, gas flow rate, and the distance between the electrodes. In this test, all parameters keep constant and only voltage value changes and are addressed its effect on the jet length. By assembling the system and testing, the breakdown occurs in a voltage of $2.5 \mathrm{kV}$ (Figure 2). From

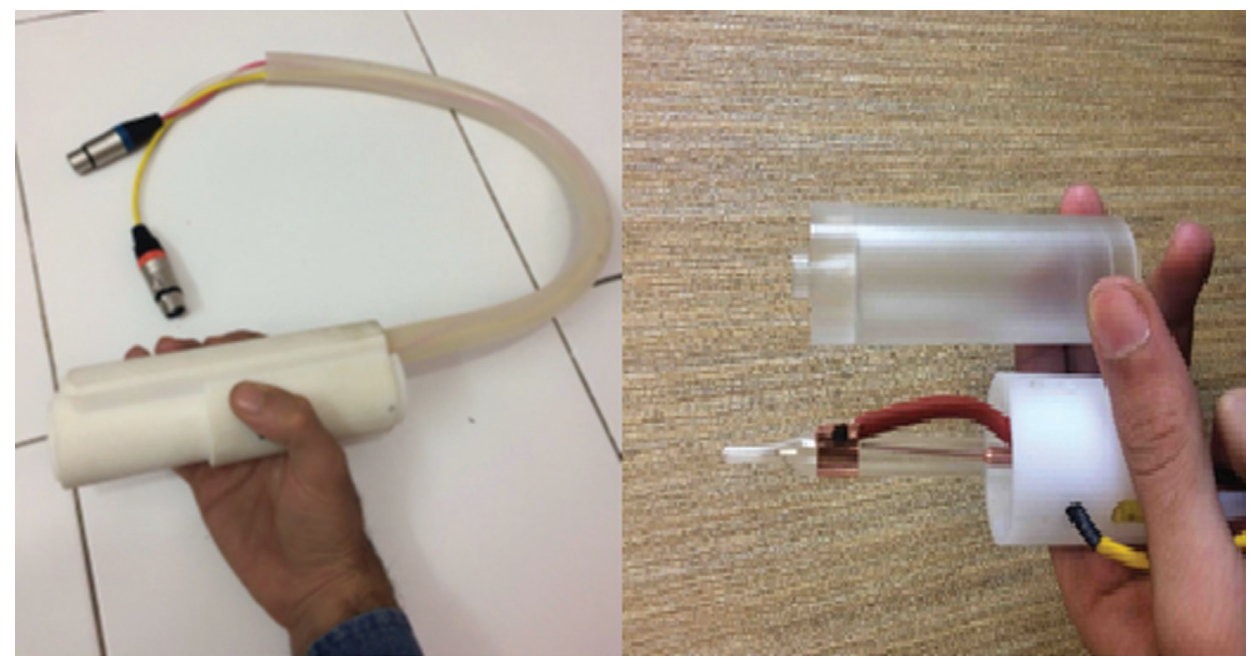

Figure 1. The manufactured handpiece (left) and its cathode and anode of plasma core (right) 

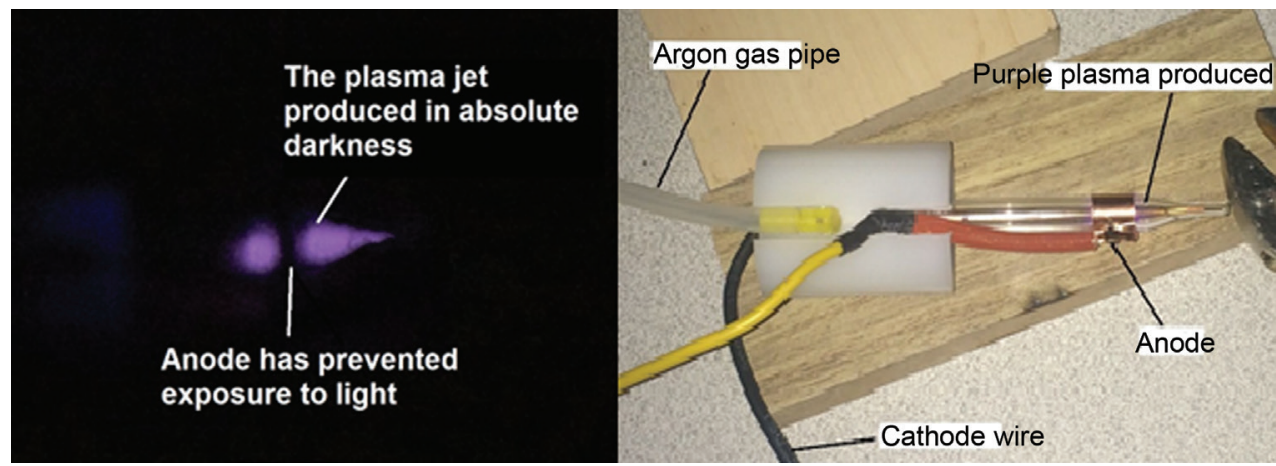

Figure 2. The gas breakdown inside the nozzle

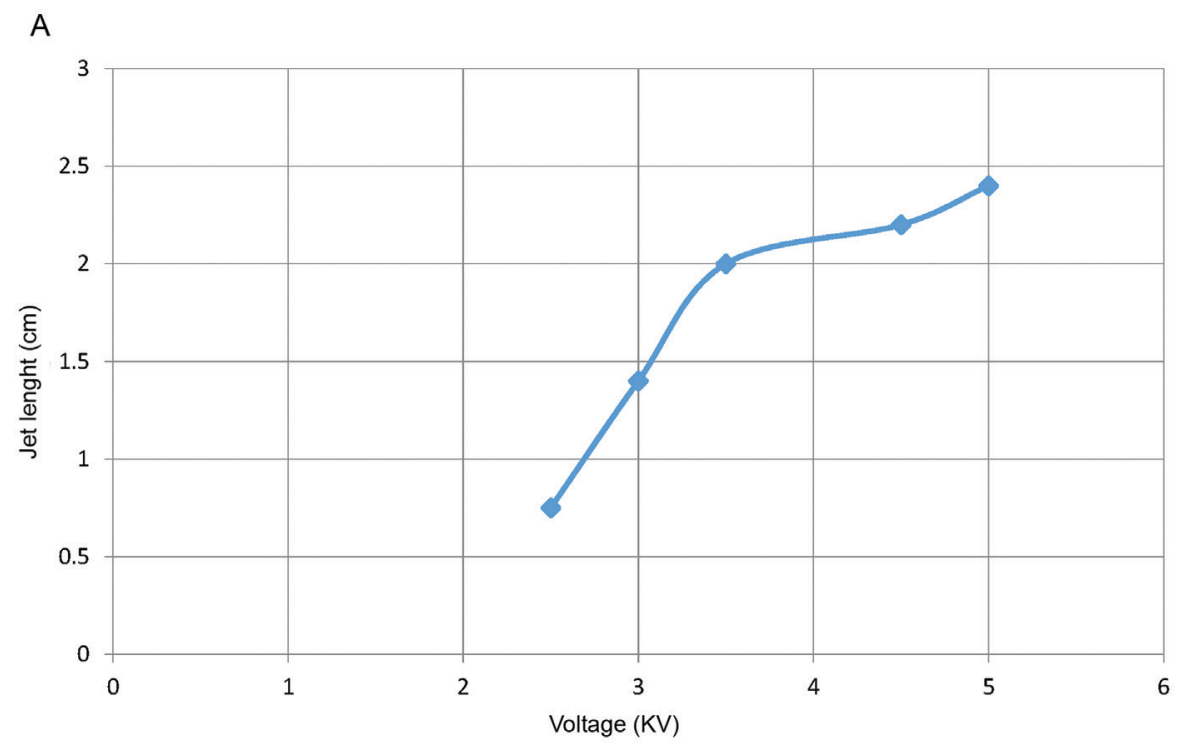

B

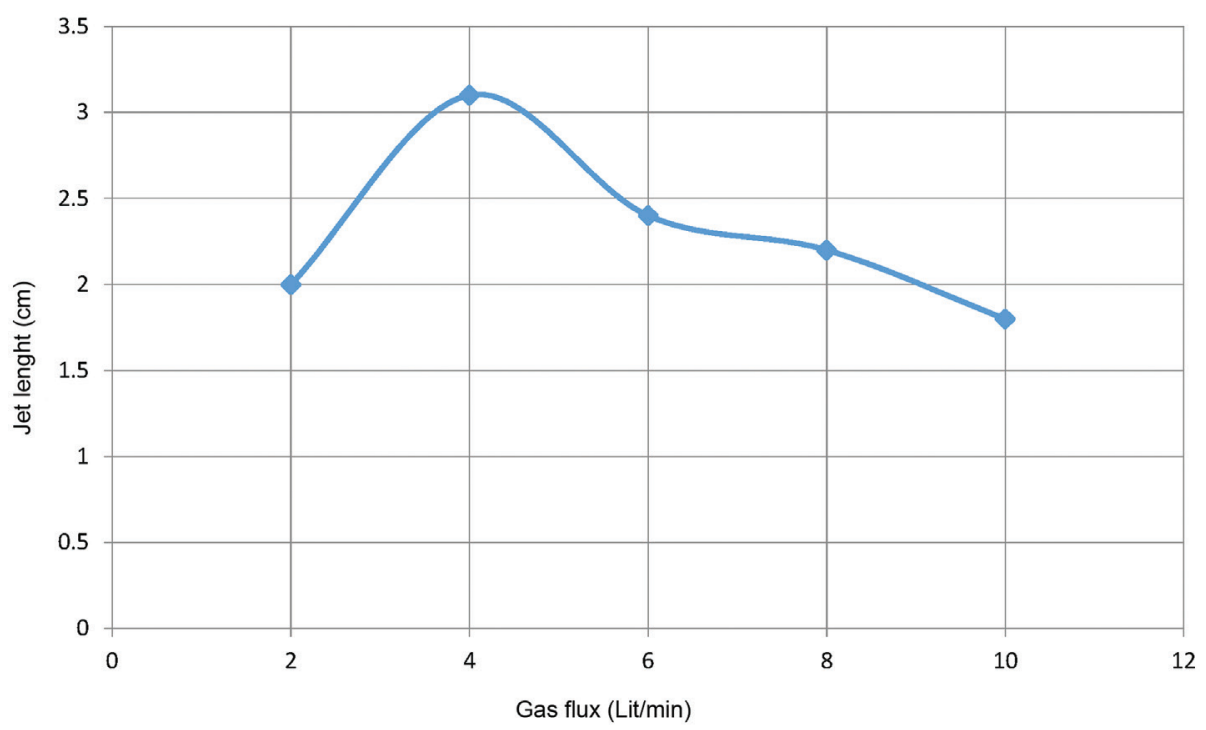

Figure 3. Graph of plasma jet length vs. voltage (A) and gas flux (B) 
observations, it is found that by increasing the voltage, the jet length increases and, in an extreme state, reaches to threshold value due to plasma density stabilization in the beam electric discharge mode (Figure 3). In the continual increasing of voltage, the plasma state turns from the beam to the arc, and the plasma converts from the cold plasma to hot plasma, which is not desirable. Gas flow is constant at $81 / \mathrm{min}$.

\section{The dependence of the jet length on the gas flux}

The amount of gas flux is an affecting factor on the plasma jet. By voltage $4.5 \mathrm{kV}$, experiments are performed to measure the effect of the gas flux on the plasma jet. Starting by the zero for gas flow, with the gradual increase in the velocity of gas flow, it is shown the jet length reaches to the threshold state. This behavior can be attributed to two main factors. First, at higher velocities, excited atoms, ions, and molecules emitting photons make a long distance before relaxation due to the return of the disturbed system to balance state. Second, at higher velocities, fluid pressure also decreases; as a result, the free mean distance increases slightly and leads to an increase in the energy relaxation length. Both processes lead to an increase in the plasma jet length (Figure 3). On the other hand, as the velocity increases, flame and jet length decreases sharply due to high losses of plasma, including ions and electrons. The results of the tests showed that by increasing the rate of argon gas flux, its concentration decreases. By a very high increase of gas flux, the jet length reduces because the plasma will not have the opportunity to recover between the electrodes and the gas flow will be turbulent.

\section{Conclusion}

In this work, the characteristics of an atmospheric plasma jet were first introduced. Then, the handpiece that was fitting this plasma was designed. The lightweight and ergonomic design, insulating against the high-voltage current, durable and beautiful body, was analyzed. After construction, it has been explained that jet length is one of the important factors in the processing of surfaces. The atmospheric plasma jet length depends on factors such as the electrodes voltage and the exhaust gas intensity. In this study, failure occurred at a voltage of $2.5 \mathrm{kV}$. Further, the flame length increased with increasing voltage, and due to stabilizing, the plasma density in a beam discharge mode, the jet length threshold occurred in particular voltage value. As more applied voltage, the plasma mode changed and turned from beam to arc. It was observed that with the increase in the voltage, jet length increased. It was also observed that with the increase in the velocity of the gas flow, the jet length reached the threshold state. This behavior can be attributed to two main factors. First, at a higher velocity, excited atoms, ions, and molecules emitting photons, travel longer distances before relaxation. At higher velocities, the fluid pressure also decreased, and as a result, the free distance increased slightly and led to increasing the energy's relaxation length. Both of these processes led to an increase in the plasma jet length. On the other hand, with increasing velocity, the flame, and jet lengths decreased greatly due to high losses of plasma, including ions and electrons. The results showed that with increasing the velocity of argon gas, its concentration decreased. This study concluded based on the result achieved, the performance of the proposed design is successful. The advantages include low-cost manufacturing, highly stable performance, and low erosion; they can make considerations for future development.

Author contributions. HB and RR contributed substantially to the conception and design of this study. RR and MK contributed substantially to the acquisition of data. All the authors analyzed and interpreted the data. RR and MK drafted the manuscript. All the authors critically revised it and approved the final version submitted for publication and take responsibility for the statements made in the published article.

Acknowledgments. We did not receive any specific grant for this research from any funding agency in the public, commercial, or not-for-profit sectors. Also, we would also like to show our gratitude to Professor Raoul Yarahmadi, Iran University of Medical Sciences, for sharing his knowledge and experience with us during the course of this research.

Conflict of interest statement. The authors have completed and submitted the International Committee of Medical Journal Editors Uniform Disclosure Form for Potential Conflicts of Interest. None of the authors disclose any conflict of interest.

\section{References}

[1] Reiazi R, Akbari ME, Norozi A, Etedadialiabadi M. Application of cold atmospheric plasma (CAP) in cancer therapy: a review. Int J Cancer Manage. 2017; 10:e8728.

[2] Laroussi M. Low-temperature plasma jet for biomedical applications: a review. IEEE T Plasma Sci. 2015; 43:703-12.

[3] Ulrich C, Kluschke F, Patzelt A, Vandersee S, Czaika VA, Richter H, et al. Clinical use of cold atmospheric pressure argon plasma in chronic leg ulcers: a pilot study. J Wound Care. 2015; 24:196, 198-200, 202-3. 


\section{Allli ASIAN BIOMEDICINE}

[4] Wende K, Bekeschus S, Schmidt A, Jatsch L, Hasse S, Weltmann KD, et al. Risk assessment of a cold argon plasma jet with respect to its mutagenicity. Mutat Res Genet Toxicol Environ Mutagen. 2016; 798-99:48-54

[5] Isbary G, Shimizu T, Li YF, Stolz W, Thomas HM, Morfill GE, Zimmermann JL. Cold atmospheric plasma devices for medical issues. Expert Rev Med Devices. 2013; 10:367-77.

[6] Guerrero-Preston R, Ogawa T, Uemura M, Shumulinsky G, Valle BL, Pirini F, et al. Cold atmospheric plasma treatment selectively targets head and neck squamous cell carcinoma cells. Int J Mol Med. 2014; 34:941-6.

[7] Walsh JL, Kong MG. Room-temperature atmospheric argon plasma jet sustained with sub-microsecond high-voltage pulses. Appl Phys Lett. 2007; 91:221502.

[8] Robert E, Sarron V, Darny T, Riès D, Dozias S, Pouvesle JM, et al. Rare gas flow structuration in plasma jet experiments. Plasma Sources Sci Technol. 2014; 23:5.

[9] Hosseinzadeh Colagar A, Memariani H, Sohbatzadeh F, Valinataj Omran A. Nonthermal atmospheric argon plasma jet effects on Escherichia coli biomacromolecules. Appl Biochem Biotechnol. 2013; 171:1617-29.
[10] Ladislav B, Baránková H. Cold atmospheric plasma: sources, processes, and applications. Thin Solid Films. 2010; 518:6705-13.

[11] Keidar M, Shashurin A, Volotskova O, Ann Stepp M, Srinivasan P, Sandler A, Trink B. Cold atmospheric plasma in cancer therapy. Phys Plasmas. 2013; 20:1-8.

[12] Case A, Messer S, Brockington S, Wu L, Witherspoon FD, Elton R, Merging of high-speed argon plasma jets. Phys Plasmas. 2013; 20:012704.

[13] Sohbatzadeh F, Hossienzadeh Colagar A, Mirzanejhad S, Motallebi S, Farhadi M, Bagheri M, et al. Design and construction of triplet atmospheric cold plasma jet for sterilization. Int J Psychol Res. 2014; 13:363-74.

[14] Mirpour S, Ghomi H, Piroozmand S, Nikkhah M, Tavassoli SH, Azad SZ, The selective characterization of a non-thermal atmospheric pressure plasma jet on the treatment of human breast cancer and normal cells. IEEE T Plasma Sci. 2014; 2:315-22.

[15] Sohbatzadeh F, Hosseinzadeh Colagar A, Mirzanejhad S. Hajiahmadi S, Talebzadeh M, Ghasemi M. Investigation of remote atmospheric pressure plasma effect on Candida albicans fungus. In: Proceedings of 30th ICPIG, 2011 August 28th - September 2nd, Belfast, Northern Ireland; 2011. 